\title{
Teeth and central nervous system: what happens when you go to Sleep
}

\begin{abstract}
In this work we will outline some unknown aspects of the teeth functions, details of their innervations and its relationship with cognitive performance and what happens when the teeth are lost. We will address the relationship between teeth and sleep and will explain how the teeth activate the ascending reticular activator system (ARAS) nuclei and allow the cerebral cortex to respond to any environmental or physiological needs even when sleep is profound. This infers that teeth are an important structure not only for chewing or the smile but also for a better functioning of our central nervous system (CNS); and explaining this correlation, between teeth and brain, is the object this paper. It appears that without teeth there is a great loss of cognite functions. The tie between the mouth and the brain is the mesencephalic trigeminal nucleus (Me5): a unique nerve formation as it contains the cell bodies of primary afferent sensory neurons; it is the only site of intra-neuraxial ganglion.
\end{abstract}

Keywords: teeth, Me5, ARAS, sleep, CAP, GABA
Volume I Issue I - 2017

\section{Giorgia Andrisani}

Andrisani Dental Clinic, Italy

Correspondence: Giorgia Andrisani, Sint Sebastiaaansbrug 23, 26II DN, Delft, Netherlands, Tel 003 |644|48385,

Email giorgia.andrisani@gmail.com

Received: April 20, 2017| Published: August 24, 2017

\section{Background}

The Me5 is a narrow band of cells that passes immediately next to the periaqueductal gray (PAG) and extends from the border between the Pons Varolii and midbrain to the superior colliculus (upper limit of the midbrain). Its lower limit is rostral to the motor trigeminal nucleus (Mo5). Next to Me5, more medially and in front of the fourth ventricle is the locus coeruleus (LC), the main source of noradrenergic fibres in the nervous system. ${ }^{1}$ The LC and Me5 are intimately related in early development; the $\mathrm{LC}$ is important in the differentiation of Me 5 neurons that are necessary for proper LC function. ${ }^{2}$ As shown in Figure 1 the Me5 is completely surrounded by the reticular formation (RF), and its caudal portion is its core consisting of small, multipolar, fusiform GABAergic cells that are rostral to the Mo5 and which we denote as Me 5 c (caudal portion of the mesencephalic trigeminal nucleus). ${ }^{3,4}$ Conversely, the rostral portion sits in the midbrain and is comprised of large pseudounipolar glutamatergic cells without dendrites. ${ }^{5-8}$ Me5 cells have gap junctions that form somatosomatic synapses among small groups of neurons..$^{9-11}$ Each neuron is almost entirely covered with processes that radiate from two or more astrocytes (usually astrocytes cover more neurons). ${ }^{12}$ The peripheral branches of mesencephalic trigeminal nucleus neurons mainly innervate mechanoreceptors in the periodontal ligament. These receptors are remarkably sensitive to movement of elevator muscles in the jaw; they are activated by a simple touch of the teeth, only a very small force is needed $(1 \mathrm{~N}$ and $4 \mathrm{~N}$ in the anterior teeth and posterior teeth, respectively). ${ }^{12-15}$ Moreover, Me5 periodontal mechanoreceptors may "fire" in the absence of stimuli. ${ }^{16}$

In humans, the stimulation of the teeth evokes sensations of touch, pressure and pain. The forces acting on the tooth, involve a movement of the element in the alveolus, induce tension in the periodontal ligament and cause the excitation of mechanoreceptors. These receptors, mechanoreceptors periodontal, are scattered in the tissues such as gingiva, cementum, periodontal ligament and alveolar bone, the receptors arranged to surround the apex, are more frequently subjected to intense and prolonged stimuli. Morphologically we distinguish three types of nerve endings: free, Ruffin-like corpuscles and Pacini-like lamellar corpuscles. The receptors are innervated by afferent fibres whose cell body lies or in the ganglion of Gasser (TG), or in Me5. Recordings affected in the Me5 nucleus have shown the presence of neurons that respond to solicitations of teeth, hard palate, and the opening of the mouth. ${ }^{17-19}$ The receptors belonging to the Gasser ganglion (TG) and those relating to Me5 (Me5) have a different topographic distribution, with Me5 concentrated around the root tip. As we have seen the force required to activate the receptors of the nerve endings of Me5 is minimal ( $1 \mathrm{~N}$ for the front teeth and $4 \mathrm{~N}$ for the back). Most of the peripheral fibres of the Me5 neurons run in the mandibular branch of the trigeminal. A collateral branch of the fibres of these neurons project directly, through the trigeminal mesencephalic tract, to the trigeminal motor nucleus. Within this arc reflex originates the masseteric reflex that determines the contraction of the masseter muscles in response to a pressure exerted on the mandibular teeth or to the lowering of the lower jaw. The central branches go to the motor trigeminal nucleus, reticular parvocellular area, and dorsolateral midbrain RF (dorsal raphe nucleus [DRN] and lateral dorsal nucleus of the tegmentum [LDT]). When they descend through the Varolio's bridge as the "stretch of Probst," they cross the dorsolateral bulb at the level of the dorsal nucleus of the vagus nerve, pass into the lateral tegmental field, and terminate in the spinal cord at the level of the caudate trigeminal nucleus up to $\mathrm{C} 1 / \mathrm{C} 3{ }^{20}$ Passing through the large neurons of the nucleus Probst, located ventrally to the solitary tract, they reach the hypoglossal nucleus, trigeminal main sensory and caudate nucleus, solitary nucleus, and eventually the supratrigeminal nucleus. ${ }^{21}$

The nerve cells of the midbrain's RF, in particular those of the DRN and LDT, both directly and through the thalamus, send 
widespread a specific projections to the entire cortex and form part of the ARAS. The Me 5 provides glutamatergic signaling to Mo5 via its large pseudounipolar neurons and GABAergic signaling from its small Me $5 \mathrm{c}$ neurons. Some branches of Me 5 carry on up towards the higher colliculi and to the nuclei of the hypothalamus orexinergic. ${ }^{22-24}$

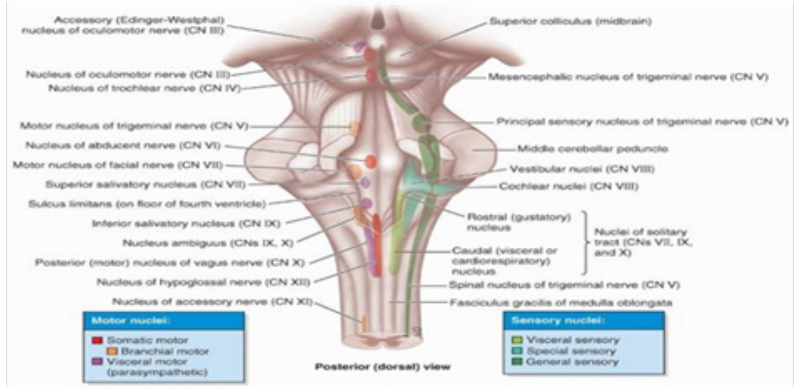

Figure I Posterior (dorsal) view.

\section{Introduction}

Every time we open or close the mouth and the teeth are touching, the Me5 is activated and activates some nuclei of ARAS, in particular those orexinergic and cholinergic, in this way actively participating in the nonspecific activation of the cerebral cortex. We can verify these claims through studies carried out on the mastication of chewing gum during an intelligence test; they show that the forced activation of the Me5 nerve endings leads to an increase in intelligence, meant as improved performance, accuracy, memory etc.. ${ }^{25-28}$ Vice versa tooth loss leads to a decrease in cognitive performance..$^{29-36}$ But in what manner Me5 exerts its action on the CNS? As we have already said, every time that the teeth touch the Me5 is activated it activates some nuclei of ARAS, and participate in the nonspecific activation of the cerebral cortex; this facilitate all the process that are happening.

The absence of teeth in edentulous, subtracts to our SNC this property making the cortex deprived of a part of nonspecific activation, so less ready to respond to any solicitation. The lack of or poor response to environmental stimuli not only makes less effective the response of our SNC but also involves its deterioration due to the LTD phenomenon (long-term depression) that leads to a progressive decrease in the number of synapses. Conversely when the Me 5 is active and effective, the LTP (long term potentiation) increases the number of synapses making our SNC more efficient. Bruxism is the moment in which the teeth touch each other with greater force and, therefore, is the time in which the Me5 is more active but bruxism is especially nocturnal: it is effective in a time of day in which, apparently, no particular "intelligence" is needed; rather it is the moment when our SNC works at its minimum, especially in the NREM stage.

\section{What thus the Me5 doing during sleep?}

Hypothesis: According to actual literature sleep bruxism is a parafunction and needs to be treated as such; we think that sleep bruxism has a more profound effect on the brain: it is responsible to maintain a constant level of arousal during sleep that can be used by the brain to react to unexpected situations. Sleep is, according to the classification dell'AASM (American Academy of Sleep Medicine; Schulz 2008), an active phenomenon, has a well defined temporal sequence, macro-structurally characterized by alternating between NREM sleep or slow or synchronized and REM sleep or rapid or desynchronized. In humans NREM sleep is divided into three different stages: N1, N2, N3. Each stage is distinguished by a greater degree of depth and a different brain activity. Already in 1930 von Bursar, studying patients with encephalitis accompanied by insomnia, he had suggested the presence of a "sleep center" at the hypothalamic level, particularly in the preoptic area/basal forebrain (30-von Bursar, 1930). In the preoptic area, there are two essential nuclei for the creation of NREM sleep: the ventrolateral preoptic nucleus (VLPO) and the median preoptic area (MNPO). The neurons of these two nuclei use inhibitory neurotransmitters such as GABA and galanin $(\mathrm{Gal})$, to stimulate sleep. ${ }^{37}$ In promoting sleep are also involved other substances such as adenosine, Substance P, interleukin 1 beta, RFGH, Prostaglandins etc.; but the most important neurotransmitter remains the GABA of hypothalamic production. ${ }^{38,39}$ With the increase of GABA decreases the production of Ach, Histamine, 5-HT, orexin, NA etc., ${ }^{40,41}$ and when the GABA level is quite high, is triggered an intrinsic mechanism in the brainstem: the of Me5.

The Me 5 is constituted for the most part from pseudounipolar cells, devoid of dendrites and, therefore, insensitive to GABA (they are also coated with several layers of glial cells that improve the isolation from the action of any other neurotransmitters); its caudal termination (Me5c), however, is made up of small multipolar cells, typically GABAergic that, at the beginning of sleep, inhibit Mo5 (Me5c the cells are situated opposite to those of the Mo5). When the GABA level is high enough it inhibits Me5c and Mo5 is uninhibited; masticatory muscles contract, in some cases so intensely as in RMMA (Rhythmic masticatory Muscle Activity), a fairly common event especially during NREM sleep; the teeth that are touching activates even more Me5 that, in turn, actives the ARAS nuclei always maintaining a certain degree of activation of these nuclei and, of course, of the cerebral cortex (arousal). Meanwhile the GABA de-activates the ARAS, the nonspecific activation; at the same time the Me5 produces a cyclic activation such as to allow us respond to any physiological or enviroinmental needs. In addition to the macro-structural sleep stages NREM and REM, a microstructure has been identified within the NREM stage by means of an electroencephalogram (EEG). This microstructure consists of alternative fluctuations characterized by sleep "superficialization". This kind of EEG tracing is called CAP (cycling alternating pattern) and can continue to occur for either short or long periods and can be interrupted by periods of normal EEG activity, which is organized according to the characteristics of that macro-structural phase (non-CAP tracing or NCAP). As shown in Figure 2, each CAP cycle is formed by alternating sequences of two kinds of EEG patterns called A-phase and B-phase. The A-phase is considered an activation phase that includes either high-voltage slow waves or low-voltage fast waves. It occurs with a frequency of about 10 seconds and makes up about $40 \%$ of the entire cycle. This phase is characterized by temporary increases in the level of consciousness, muscle tone, and vegetative functions and represents the expression of activation of neuronal systems during sleep.

\section{The CAP A-phase is divided into three different subtypes: $\mathrm{A} 1, \mathbf{A} 2$, and $\mathbf{A} 3$}

Functionally, CAP represents an index of the arousal level that goes from a high (A-phase) to a low arousal condition (B-phase). This instability may be due to various individual internal and external factors. This instability of arousal is associated with a corresponding activation of various polysomnographic (PSG) parameters (EEG, muscle tone, cardio respiratory frequency) during the A-phase. However, all these parameters return to their baseline values during 
the B-phase. The existence of a "permissive window" during NREM sleep has been hypothesized, which may occur during the A2 and A3 sub-types to facilitate the occurrence of pathological events. On the other hand, the B-phase may work to oppose such events. This process is defined as the gating effect and has been reported in literature relating to various types of sleep disorders including bruxism, ${ }^{42}$ periodic limb movement (PLM), obstructive sleep apnea syndrome (OSAS), epileptic anomalies, and insomnia. ${ }^{43-45}$ The B-phase, on the other hand, has been closely associated to sleep apnea, with breathing returning to normal in the A-phase. ${ }^{46}$

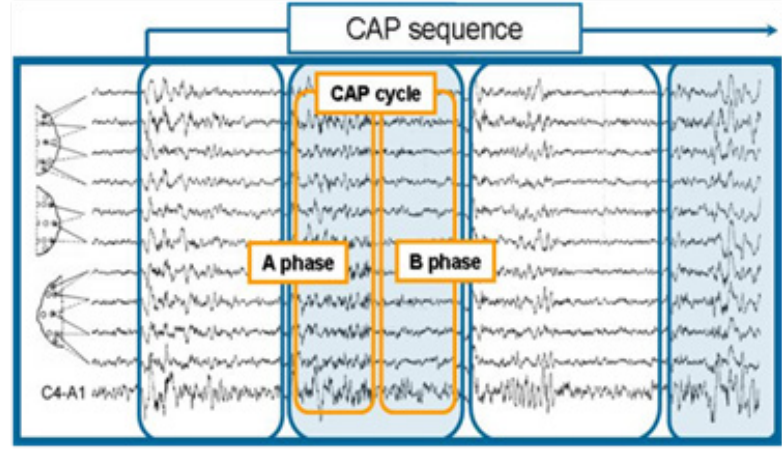

Figure 2 CAP sequences.

\section{Why do the A phases of CAP allow for these pathological events?}

The difference between the A and B phases is that in the latter, the GABA inhibitory effect prevails in the brainstem, while the former is characterized by an increase in levels of ARAS neurotransmitters, mainly NA and Ach. These neurotransmitters stimulate the cerebral cortex, resulting in an increase in arousal, as shown by EEG recordings in phase A of CAP. In every CAP phase, especially those with the A2 and A3 subtypes, there are periods of very high-frequency EEG and sympathetic nervous system activity. At the end of these episodes, EMG tracing sometimes reveals rhythmic masticatory muscle activity (RMMA) with an average frequency of 1.8 occurrences per sleep hour in non-bruxism sleepers. ${ }^{47,48}$ RMMA occurrences are the PSG representations of sleep bruxism (SB), but are not associated with teeth grinding, orofacial pain, or other disorders. Most RMMA occurrences are observed in the NREM phase, with fewer occurring in the REM phase For example, in one study, which analyzed $69 \%$ of SB episodes, $6 \%$ occurred in phase N1, $64 \%$ in phase $\mathrm{N} 2,10 \%$ in phase $\mathrm{N} 3$, and $21 \%$ occurred during REM sleep. ${ }^{49}$

\section{Discussion}

\section{Approximately $\mathbf{8 5} \%$ of SB episodes occur in a specific order}

In the 4-8 minutes preceding muscle activation, there is an increase in sympathetic autonomic activity in the heart caused by a decrease in the parasympathetic effect. In the 4 seconds preceding muscle activation, there is an increase in high frequency cortical activity (micro-arousal, as seen in the EEG). In the last second preceding muscle activation, there is a simultaneous increase of about $25 \%$ in heart rate and of about $100 \%$ of the respiratory effort amplitude. Likewise, there is a simultaneous increase of the tone of the suprahyoid muscles and respiratory tracts. RMMA occurs following this sequence of events. SB episodes occur in clusters (75.8\%) and are characterized by micro arousals that last between 3 and 10 seconds. During this period of time, cerebral, cardiac, and respiratory activity, as well as muscle tones are regenerated and RMMA/SB occurs. RMMA episodes happen in $58 \%$ of the adult population. Therefore, RMMA is a normal physiological sleep activity, often associated with the A2 or A3 subtypes of A-phase CAP.

With a calm patient, in a protected environment (such as in a clinic) and in the absence of external stimuli, a PSG recording that reveals occurrences of CAP indicates that cortical activation is derived solely by internal stimuli. Because the majority of physiological functions are monitored by PSG and video recording without signs of activation, we assume that during clinical recording CAP cortical activation is due to the ARAS nuclei. However, these nuclei are subject to the GABA inhibitory effect. Furthermore, only the Me5 can activate the ARAS nuclei because its cells lack dendrites protection by glia cells from the effect of many neurotransmitters; in this case, GABA. ${ }^{26}$ As mentioned above, Me5 periodontal and intramuscular terminations are extremely sensitive, ${ }^{12-14}$ the Me5 periodontal mechanoreceptors may also fire spontaneously without stimuli. ${ }^{16} \mathrm{Me} 5$ cells also have electrical synapses (gap-junctions) and can form somato-somatic synapses between two neurons or among small groups of neurons. These gap-junctions are able to promote communication with other cells, inducing them to become active, thereby causing CAP. When clinical EEG and EMG recordings are simultaneously performed and show one CAP (possibly during A2 or A3) and one RMMA, it can be assumed that the two effects are related to the same event, namely activation of RMMA and more specifically, the Me5. This can be assumed because Me5 activation causes the activation of ARAS nuclei, which in turn produces arousal and therefore CAP However, a noise normally causes arousal, as indicated by a CAP in the EEG. But why should the occurrence of a CAP corresponding to a RMMA be attributed to a "permissive window" and not to the RMMA? In an unprotected environment there are combinations of CAP events due to external and internal factors in addition to Me5. However, during the protected environment of a PSG, in the absence of any patient physical activities, we can be certain that episodes of CAP are solely due to the activation of ARAS nuclei by the Me5. This is especially true when these episodes occur within the A2 and A3 subtypes which temporally correspond to the RMMA (as seen in the EMG). In a clinical environment, CAP episodes can be attributed to Me5 activity even when they do not occur at the same time as those of RMMA. In addition the Me5 nucleus is the only nucleus that is not inhibited by GABA that can also activate ARAS nuclei. No other nuclei present these two properties. Thus, the A phases of CAP that occur during PSG recording in a protected environment represent the EEG recording of Me5 activity, even in absence of RMMA due to Me5's ability to fire spontaneously. ${ }^{16}$ RMMA represents periods of high Me5 activation. CAP is then characterized by an increase of arousal (activation of the ARAS nuclei by Me5) and sympathetic activity (LC activation by Me5). Other Me5 target cells are Me $5 \mathrm{c}$ and Mo5. At regularly occurring intervals and during NREM phases N1, N2, and N3, the Me5 fires "spontaneously", releasing GLU in the ARAS, Me 5c, and Mo5 nuclei. This allows the Me5c to "contrast" GABA's inhibitory effect, making sleep deeper and more restorative by producing the higher GABA concentrations required for Me5 inhibition. Also, this mechanism allows Mo5 to be "ready" to become activated immediately after Me5c inhibition. In conclusion, the Me5 mechanism probably becomes necessary because sleep is naturally dangerous when there are high GABA concentrations, as too many 
cells become non functional, creating a potentially perilous situation. For example, sleep apnea is frequent during the $\mathrm{N}$ or B CAP phases, when GABA levels are very high and probably inhibit respiratory centres. ${ }^{50}$ Basically, if the RMMA is activated it means that Mo5 is enabled and therefore that Me $5 \mathrm{c}$ is deactivated and, since we are in the sleep phases, can happen only due to the action of GABA.

\section{Conclusion}

There are number of supporting proofs for our hypothesis. First, Me5 is present in the midbrain and not in the Gasserian ganglion (its location may be due to an evolutionary advantage related to its function). Second, it has been shown that activation of the cerebral cortex is caused by simple mastication of chewing-gum. ${ }^{50,51}$ Third, there is evidence that the use of pacifiers reduces sudden infant death syndrome (SIDS) mortality in infants. Fourth, studies have revealed the presence of the same kind of lesions found in children who died from SIDS and test subjects that underwent bilateral lesion of Me5 fibres. ${ }^{52}$

\section{Fifth and most important are the findings relating to CAP}

In physiological conditions, such as when a patient sleeps at home, CAP can be caused by many factors. However, during CAP recording in a protected environment (such as in a clinic), the occurrence of CAP can be triggered only by internal factors. In fact, when the majority of the body functions are monitored by PSG and sleep activity monitors, it can be assumed that CAP is due to the activation of one or more of the ARAS nuclei from Me5. This is further confirmed when simultaneous EEG and EMG recordings show the presence of CAP and RMMA, respectively, as often occurs with CAP with A2 and A3 subtypes. This also confirms that Me5 activation causes arousal and therefore activation of ARAS nuclei. All individuals have CAP; however, only $58 \%$ have RMMA.

In other words, the Me5 is activated in all individuals, but only in $58 \%$ of the cases does this activation occur in the manner described above, giving rise to the $\mathrm{A} 2$ and $\mathrm{A} 3$ subtypes of CAP that temporally correspond to RMMA. GABA is naturally the key of this process: when GABA levels are high, they inhibit Me5c, activating RMMA. On the other hand, low GABA levels can cause decrease in RMMA. In any case, the Me5 naturally remains active and can be responsible for the CAP A1 subtype characterized by inactivation of muscle tone and tonic touch of teeth. This activation of Me 5 stimulates the release of NA and Ach from VLPO, which are the main GABA inhibitors.

\section{Acknowledgements}

None.

\section{Conflict of interest}

The authors declare that the research was conducted in the absence of any commercial or financial relationships that could be construed as a potential conflict of interest.

\section{Ethics statement}

The study presented in the manuscript does not involve human or animal subjects.

\section{Author contribution statement}

Andrisani Giovanni: research and study of the pathology, theory development.

\section{References}

1. Rokx JT, Jüch PJ, van Willigen JD. Arrangement and connections of mesencephalic trigeminal neurons in the rat. Acta Anat (Basel). 1986;127(1):7-15.

2. Espana A, Clotman F. One cut factors control development of the locus coeruleus and of the mesencephalic trigeminal nucleus. Mol Cell Neurosci. 2012;50(1):93-102.

3. Kolta A, Westberg KG, Lund JP. Identification of brainstem inter-neurons projecting to the trigeminal motor nucleus and adjacent structures in the rabbit. J Chem Neuroanat. 2000;19(3):175-195.

4. Hayar A, Poulter MO, Pelkey K, et al. Mesencephalic trigeminal neuron responses to gamma-aminobutyric acid. Brain Res. 1997;753(1):120-127.

5. Copray JC, Liem RS, van Willigen JD. Morphological arrangement between astrocytes and trigeminal mesencephalic primary afferent neurons in the rat. Exp Brain Res. 1990;83(1):215-218.

6. Paik SK, Kwak MK, Ahn DK, et al. Ultra structure of jaw muscle spindle afferent swith in the rat trigeminal mesencephalic nucleus. Motor Systems. 2005;16(14):1561-1574.

7. Lazarov NE. Comparative analysis of the chemical neuroanatomy of the mammalian trigeminal ganglion and mesencephalic trigeminal nucleus. Prog Neurobiol. 2002;66(1):19-59.

8. Henderson G, Pepper CM, Shefner SA. Electrophysiological properties of neurons contained in the locus coeruleus and mesencephalic nucleus of the trigeminal nerve in vitro. Exp Brain Res. 1982;45(1-2):29-37.

9. Curti S, Hoge G, Nagy JI, et al. Synergy between electrical coupling and membrane proper ties promotes strong synchronizat ion of neurons of the mesencephalic trigeminal nucleus. J Neurosci. 2012;32(13):4341-4359.

10. Liem RS, Copray JC, van Willigen JD. Ultra structure of the rat mesencephalic trigeminal nucleus. Acta Anat (Basel). 1991;140(2):112119.

11. Baker R, Llinás R. Electrotonic coupling between neurones in the rat mesencephalic nucleus. J Physiol. 1971;212(1):45-63.

12. Trulsson M. Sensory-motor function of human periodontal mechanoreceptors. J Oral Rehabil. 2006;33(4):262-273.

13. Trulsson M. Force encoding by human periodontal mechanoreceptors during mastication. Arch Oral Biol. 2007;52(4):357-360.

14. Trulsson M, Gunne HS. Food-holding and biting behavior in human subjects lacking periodontal receptors. J Dent Res. 1998;77:574-582.

15. Nishigawa K, Bando E, Nakano M. Quantitative study of bite force during sleep associated bruxism. J Oral Rehabil. 2001;28(5):485-491.

16. Trulsson M, Johansson RS, Olsson KA. Directional sensitivity of human periodontal mechano-receptive afferents to forces applied to the teeth. $J$ Physiol. 1992;447:373-389.

17. Passatore M, Filippi GM. Sympathetic modulation of periodontal mechanoreceptors. Arch Ital Biol. 1983;121(1):55-65.

18. Passatore M, Lucchi ML, Filippi GM, et al. Localization of neurons innervating masticatory muscle spindle and periodontal receptor $\mathrm{s}$ in the mesencephalic trigeminal nucleus and their reflex actions. Arch Ital Biol. $1983 ; 121(2): 117-130$ 
19. Cash RM, Linden RW. Effects of sympathetic nerve stimulation on intraoral mechanoreceptor activity in the cat. J Physiol. 1982;329:451-463.

20. Matsushita M, Okado N, Ikeda M, et al. Descending projections from the spinal and mesencephalic nuclei of the trigeminal nerve to the spinal cord in the cat. A study with the horseradish peroxidase technique. J Comp Neurol. 1981;196(2):173-187.

21. Matesz C. Peripheral and central distribution of fibres of the mesencephalic trigeminal root in the rat. Neurosci Lett. 1981;27(1):13-17.

22. Ndiaye A, Pinganaud G, VanderWerf F, et al. Connections between the trigeminal mesencephalic nucleus and the superior colliculus in the rat. Neurosci Lett. 2000;294(1):17-20.

23. Yokoyama S, Kinoshita K, Muroi Y, et al. The effects of bilateral lesions of the mesencephalic trigeminal sensory nucleus on nocturnal feeding and related behaviours in mice. Life Sci. 2013;93(18-19):681-686.

24. Ishii T1, Suenaga R, Iwata W, et al. Bilateral lesions of the mesencephalic trigeminal sensory nucleus stimulate hippocampal neurogenesis but lead to severe deficits in spatial memory resetting. Brain Res. 2010;1342:74 84.

25. Hirano Y, Onozuka M. Chewing and cognitive function. Brain Nerv. 2014;66(1):25-32.

26. Hirano Y, Obata T, Takahashi H, et al. Effects of chewing on cognitive processing speed. Brain Cogn. 2013;81(3):376-381.

27. Smith A. Effects of chewing gum on mood, learning, memory and performance of an intelligence test. Nutr Neurosci. 2009;12(2):81-88.

28. Morgan $\mathrm{K}$, Johnson AJ, Miles C. Chewing gum moderates the vigilance decrement. Br J Psychol. 2014;105(2):214-225.

29. Mayor S. Gum disease is an early sign of diabetes, and tooth loss is associated with risk of dementia, studies find. BMJ. 2017;356:j1225.

30. Takeuchi K, Ohara T, Furuta M, et al. Tooth loss and risk of dementia in the community: the Hisayama study. J Am Geriatr Soc. 2017;65(5):e95100 .

31. Tonsekar PP, Jiang SS, Yue G. Periodontal disease, tooth loss and dementia: Is there a link? A systematic review. Gerodontology. 2017;34(2):151-163.

32. Shen T, Lv J, Wang L, et al. Association between tooth loss and dementia among older people: a meta-analysis. Int J Geriatr Psychiatry. 2016;31(8):953-935.

33. Felton DA. Complete edentulism and comorbid diseases: an update Felton DA. J Prosthodont. 2016;25(1):5-20.

34. Luo J, Wu B, Zhao Q, et al. Association between tooth loss and cognitive function among 3063 Chinese older adults: a community-based study. PLoS One. 2015;10(3):e0120986.

35. Stewart R, Stenman U, Hakeberg M, et al. Associations between oral health and risk of dementia in a 37-year follow-up study: the prospective population study of women in Gothenburg. J Am Geriatr Soc. 2015;63(1):100-105.

36. Zhu J, Li X, Zhu F, et al. Multiple tooth loss is associated with vascular cognitive impairment in subjects with acute ischemic stroke. J Periodontal Res. 2015;50(5):683-688.
37. Saper CB, Chou TC, Scammell TE. The sleep switch: hypothalamic control of sleep and wakefulness. Trends Neurosci. 2001;24(12):726-731.

38. Luppi PH. Neurochemical aspects of sleep regulation with specific focus on slow-wave sleep. World J Biol Psychiatry. 2010;11(Suppl 1):4-8.

39. Szymusiak R1, McGinty D. Hypothalamic regulation of sleep and arousal. Ann N Y Acad Sci. 2008;1129:275-286.

40. Sherin JE, Elmquist JK, Torrealba F, et al. Innervation of histaminergic tuberomammillary neurons by GABAergic and galaninergic neurons in the ventrolateral preoptic nucleus of the rat. J Neurosci. 1998;18(12):47054721

41. Hsieh KC, Gvilia I, Kumar S, et al. c-Fos expression in neurons projecting from the preoptic and lateral hypothalamic areas to the ventrolateral periaqueductal gray in relation to sleep states. Neuroscience. 2011;188:55-67.

42. Macaluso GM, Guerra P, Di Giovanni G, et al. Sleep bruxism is a disorder related to periodic arousals during sleep. J Dent Res. 1998;77(4):565-573.

43. Parrino L, Smerieri A, Spaggiari MC, et al. Cyclic alternating pattern (CAP) and epilepsy during sleep: how a physiological rhythm modulates a pathological event. Clin Neurophysiol. 2000;111(Suppl 2):S39-46.

44. Halász P, Terzano MG, Parrino L. Spike-wave discharge and the microstructure of sleep-wake continuum in idiopathic generalized epilepsy. Neurophysiol Clin. 2002;32(1):38-53.

45. Parrino L, Boselli M, Buccino GP, et al. The cyclic alternating pattern plays a gate-control on periodic limb movements during non-rapid eye movement sleep. J Clin Neurophysiol. 1996;13(4):314-323.

46. Terzano MG, Parrino L, Boselli M, et al. Polysomnographic analysis of arousal responses in obstructive sleep apnea syndrome by means of the cyclic alternating pattern. J Clin Neurophysiol. 1996;13(2):145-155.

47. Carra MC1, Rompré PH, Kato T, et al. Sleep bruxism and sleep arousal: an experimental challenge to assess the role of cyclic alternating pattern. J Oral Rehabil. 2011;38(9):635-642.

48. Zucconi M, Oldani A, Ferini-Strambi L, et al. Arousal fluctuations in nonrapid eye movement parasomnias: the role of cyclic alternating pattern as a measure of sleep instability. J Clin Neurophysiol. 1995;12(2):147-154.

49. Huynh N, Kato T, Rompré PH, et al. Sleep bruxism is associated to microarousals and an increase in cardiac sympathetic activity. J Sleep Res. 2006;15(3):339-346.

50. Morgan K, Johnson AJ, Miles C. Chewing gum moderates the vigilance decrement. Br J Psychol. 2014;105(2):214-225.

51. Lexomboon D1, Trulsson M, Wårdh I, et al. Chewing ability and tooth loss: association with cognitive impairment in an elderly population study. $J$ Am Geriatr Soc. 2012;60(10):1951-1956.

52. Andrisani G, Andrisani G. The mesencephalic nucleus of the trigeminal nerve and the SIDS. Med Hypotheses. 2015;84(1):8-10. 